\title{
Outcomes in Adolescents Undergoing Treatment for Drug-Resistant Tuberculosis in Mumbai
}

\author{
Petros Isaakidis ${ }^{1, *}$; Mrinalini Das ${ }^{1}$; Peter Saranchuk ${ }^{2}$ \\ ${ }^{1}$ Médecins Sans Frontières (MSF)/Doctors Without Borders, Mumbai, India \\ ${ }^{2}$ Southern Africa Medical Unit (SAMU), Médecins Sans Frontières, Cape Town, South Africa \\ ${ }^{*}$ Corresponding author: Petros Isaakidis, Médecins Sans Frontières (MSF)/Doctors Without Borders, Mumbai, India. Tel: +91-9930534211, Fax: +91-2226462769, \\ E-mail: msfocb-asia-epidemio@brussels.msf.org
}

Received: May 30, 2015; Accepted: May 31, 2015

Keywords: Adolescent; HIV; Outcomes; Tuberculosis, Multidrug-Resistant; Mumbai

\section{Dear Editor,}

We appreciate the report by Moyo et al. on adolescents undergoing treatment for drug-resistant tuberculosis (DR-TB) in South Africa (1). The poor outcomes in their adolescent cohort, the largest reported to-date, were unfortunately comparable to the outcomes we had earlier reported among adolescent DR-TB patients co-infected with HIV in Mumbai (2). However, we would like to share some encouraging new experience regarding the treatment of adolescents (10 - 19 years) with DR-TB in Mumbai. Between July 2007 and April 2015, we enrolled 47 adolescent DR-TB patients with a median (IQR) age of 15 (13 - 18) years. Thirty-three (70\%) of them were female and $28(60 \%)$ were co-infected with HIV. This was a cohort with complex resistance patterns: 3 patients had polyresistant TB, 14 had MDR-TB (defined as resistance to rifampicin and isoniazid), 17 had pre-XDR-TB (MDR-TB with additional resistance to a fluoroquinolone or an injectable drug), 8 had XDR-TB (MDR-TB with additional resistance to both a fluoroquinolone and an injectable drug) and one had XXDR-TB (XDR-TB with additional resistance to a Group 4 anti-TB drug). For four patients, the diagnosis was not bacteriologically confirmed, but was based on clinical symptoms and history of contact with a confirmed case. Patients were referred to us from public and public-private antiretroviral-treatment (ART) centers and from a network of community non-governmental organizations. In early 2014, we received a cluster of cases referred to our clinic from a girl-only orphanage. After conducting an outbreak investigation and systematic contact-tracing in the orphanage and the adjacent public school, we diagnosed six adolescents with DR-TB who were then enrolled in our cohort.

Of these 47 adolescents with DR-TB, three never commenced treatment: two of them died before the treat- ment initiation and one refused treatment. Of 26 adolescents with final treatment outcomes available, 13 (50\%) had a favorable outcome ( 10 were cured and 3 completed treatment), while 9 (35\%) died and 4 (15\%) were lost from the treatment. Of 18 patients still on treatment at the time of this analysis, 10 patients had completed the intensive phase of treatment and had culture conversion to negative and 8 patients were yet to complete the intensive phase, but the majority of the latter were clinically stable. As did Moyo et al. (1), we found that loss-from-treatment (relative risk (RR): 0.78, $\mathrm{P}=0.27$ ) and treatment success (cure or treatment completion) (RR: $0.71 ; \mathrm{P}=0.67$ ) did not differ between HIV-infected and un-infected adolescents. We strongly support the hypothesis of Moyo et al. (1) that delayed diagnosis rather than treatment failure was the reason for poor outcomes in South African and early Indian cohorts. Looking at significant improvement in DR-TB treatment outcomes we reported here, we further hypothesize that it may be due to earlier diagnosis. Firstly, after disappointing results of our first study, we proactively sought adolescent patients at the public ART centers and encouraged their referral to our facility where stronger patient support activities were in place. Secondly, the systematic contact tracing that was implemented after we identified the institutional DR-TB outbreak resulted in earlier diagnosis and treatment initiation. Moreover, we believe that the intensified support to adolescents that we have provided since our previous study may explain the significant drop in the proportion of patients lost-from-treatment (27\%, versus $3 \%$, P Value $<0.05$ ). Our study is limited by the small number of patients and the highly complex resistant patterns, which may not be generalizable to other settings or populations.

A better understanding of specific needs of adolescents

Copyright (C) 2015, Pediartric Infections Research Center. This is an open-access article distributed under the terms of the Creative Commons Attribution-NonCommercial 4.0 International License (http://creativecommons.org/licenses/by-nc/4.0/) which permits copy and redistribute the material just in noncommercial usages, provided the original work is properly cited. 
with DR-TB is urgently needed across the globe. In addition to strategies that allow for earlier detection of DR-TB in adolescents, we need specific, evidence-based strategies to keep adolescent DR-TB patients on treatment. In the meantime, TB-programs can borrow strategies from HIV-programs that have managed to improve adherence to ART and retention in care of adolescents living with HIV, such as community-based approaches, specific training of health care workers and other adolescent-friendly services $(3,4)$. And we need to do something about the current DR-TB treatment regimens, which are particularly challenging for adolescents to adhere to and have been described as "worse than the disease itself" by patients (5). The miserably high mortality rate among this vulnerable group gives us no other choice but immediate action.

\section{Acknowledgements}

We wish to acknowledge the staff of MSF-Mumbai clinic, the SION Center for Excellence in Pediatric HIV Care, Mumbai as well as the adolescent patients and their families.

\section{Authors' Contributions}

Study concept and design: Petros Isaakidis, Mrinalini
Das, Peter Saranchuk PI, MD, PS; Acquisition of data: Mrinalini Das; Analysis and interpretation of data: Petros Isaakidis, Mrinalini Das, Peter Saranchuk; Drafting of the manuscript: Petros Isaakidis; Critical revision of the manuscript for important intellectual content: Mrinalini Das, Petros Isaakidis; Statistical analysis: Mrinalini Das, Petros Isaakidis; Administrative, technical and material support: Petros Isaakidis; Study supervision: Petros Isaakidis.

\section{References}

1. Moyo S, J Furin J, Hughes J, Daniels J, Snyman L, Muller O, et al. Outcomes in Adolescents Undergoing Treatment for Drug-resistant Tuberculosis in Cape Town, South Africa, 2008-2013. Arch Pediatr Infect Dis. 2014;2(2):e17934

2. Wilkinson RJ, Isaakidis P, Paryani R, Khan S, Mansoor H, Manglani $\mathrm{M}$, et al. Poor Outcomes in a Cohort of HIV-Infected Adolescents Undergoing Treatment for Multidrug-Resistant Tuberculosis in Mumbai, India. PLOS ONE. 2013;8(7):e68869.

3. WHO.. HIV and adolescents: guidance for HIV testing and counselling and care for adolescents living with HIV: recommendations for a public health approach and considerations for policy-makers and managers.: World Health Organization; 2013.

4. Shroufi A, Gunguwo H, Dixon M, Nyathi M, Ndebele W, SaintSauveur J, et al. HIV-infected adolescents in southern Africa can achieve good treatment outcomes. Aids. 2013;27(12):1971-8.

5. Isaakidis P, Rangan S, Pradhan A, Ladomirska J, Reid T, Kielmann K. 'I cry every day': experiences of patients co-infected with HIV and multidrug-resistant tuberculosis. Trop Med Int Health. 2013;18(9):1128-33. 